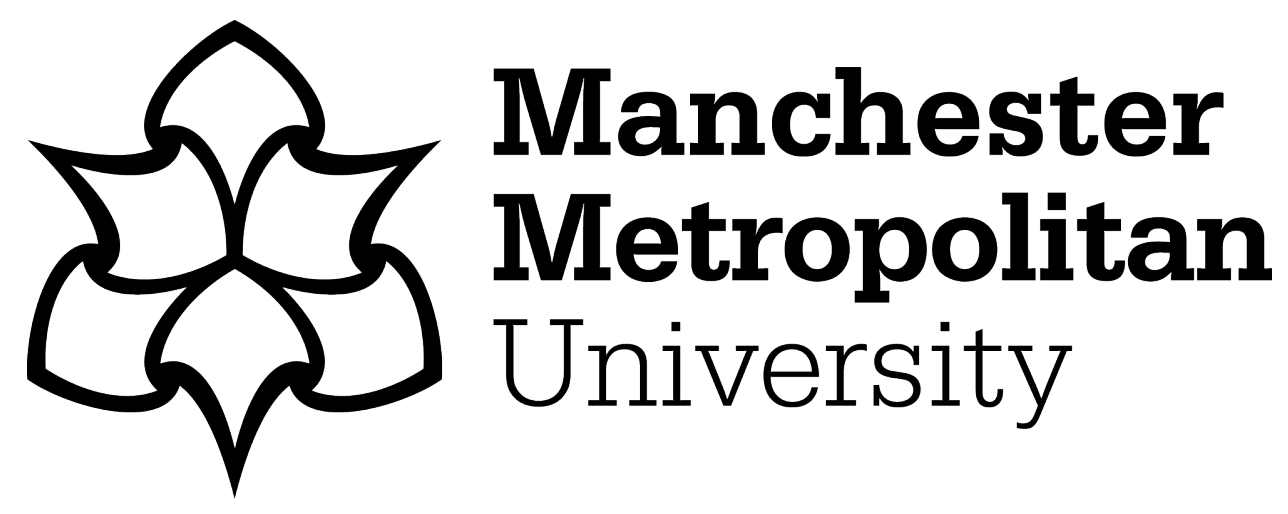

Ghasemi, Parisa and Karimian, Noushin (2020) A Qualitative Study of Various Aspects of the Application of IoT in Disaster Management. In: 2020 6th International Conference on Web Research (ICWR), 22 April 2020 - 23 April 2020, Tehran, Iran.

Downloaded from: https://e-space.mmu.ac.uk/626761/

Publisher: IEEE

DOI: https://doi.org/10.1109/icwr49608.2020.9122323

Please cite the published version 


\section{A Qualitative Study of Various Aspects of the Application of IoT in Disaster Management}

\author{
Parisa Ghasemi \\ Faculty of Engineering Science \\ University of Tehran, Tehran, \\ Parisa.Ghasemi@ut.ac.ir
}

\author{
Dr Noushin Karimian \\ Faculty of Engineering Science \\ University of Tehran, Tehran, \\ NKarimian@ut.ac.ir
}

\begin{abstract}
Considering the vital issue of disaster management and the requirement of its additional development due to the increase in the rate of disasters over the recent years, using new technologies in all stages of disaster management including preparation stages for pre-disaster periods, reaction to disaster during its occurrence as well as the post-disaster reconstruction is very important. Therefore, the capacity of IoT in creating intelligence in objects to investigate different environmental parameters, the capacity of analyzing information, the intelligence required to predict some events and consequently, responding timely to any event and even proposing programs and systems for human performance are very effective in managing disasters. The existing resources for investigating the usages of Internet of Things (IoT) in disaster management could each address a particular aspect of this application. This paper has attempted to present a comprehensive view regarding the different dimensions of application of IoT in disaster management. The advantages and disadvantages of this application are mentioned, some instances of previous systems and models have also been discussed. The novelty of this work is the extent of examined dimensions and also addressing what can be done in different kinds of disasters.
\end{abstract}

Keywords-Internet of Things (IoT), Disaster Management, Earthquake, Flood, Fire, Challenge.

\section{INTRODUCTION}

The growth and development of industry and population has caused climate changes, production of waste and pollution (thermal, chemical, radioactive, etc.) on a global scale, and consequently, the occurrence of numerous natural and man-made disasters in higher rates. This reveals the necessity of more investigation and investment for dealing with these disasters. In recent decades, disaster management has become one of the critical concerns of nongovernmental organizations as well as governments. The growth of new technologies has always changed the form of disaster management, as the tools and capacities of human beings vary based on current technology. With the appearance of IoT, many plans have been proposed and implemented in the fields of making preparations, predicting situation and controlling the post-disaster conditions. IoT can accelerate every step of disaster management process and optimize the resource allocation. Using IoT technology in disaster management has many advantages the most important of which are: (i) Ability to visualize the operations more precisely, (ii) Creation of further information resources which are faster and more accessible, (iii) Creation of the required infrastructures for the involvement of all stakeholders in disaster management, (iv) Optimization and simplification of all emergency management processes [1]. The tremendous advantages of using IoT in disaster management have provided the required motivation to examine this issue. Technologies always try to produce solutions to the real-world problems. With the emergence of any technology, many concerns and opportunities are revealed which make us examine every aspect of it. Therefore, in this paper, we have discussed and examined the various aspects of the application of IoT in disaster management. In order to examine the issue, in Section 2, the concepts of the paper's working area i.e. IoT and disaster management are briefly presented. In Section 3, the effects of using Internet of Things in each phase of disaster management are examined. In Section 4, the infrastructures and technologies required to implement practically the plans are introduced. In Section 5, the issues and challenges existing in this field are mentioned. In Section 6, the application of IoT in several types of natural disasters is described specifically and finally in Section 7, the paper is concluded.

\section{A REVIEW OF CONCEPTS}

\section{A. Internet of Things}

The concept of Internet of Things refers to the creation of awareness and understanding in the virtual or real objects that lead to their independence and decision-making in different situations as well as the capacity to connect and cooperation between them to make joint decisions (if needed) which happens by receiving information from the sensors and then, analyzing them or sending them to other nodes of the network [2].

Although it is only two decades since we know this technology with its current title, this objective has been lasted for a long time; even before the official introduction of the Internet of Things, its significant implementations have been existed. Here, the highlighted points of the Internet of Things development trend, which has a direct effect on its use in disaster management, are illustrated in the Fig. 1.

In recent years, IoT has been followed more seriously and has been used in all areas of technology and everyday life as well as industries, as such the existence of IoT in

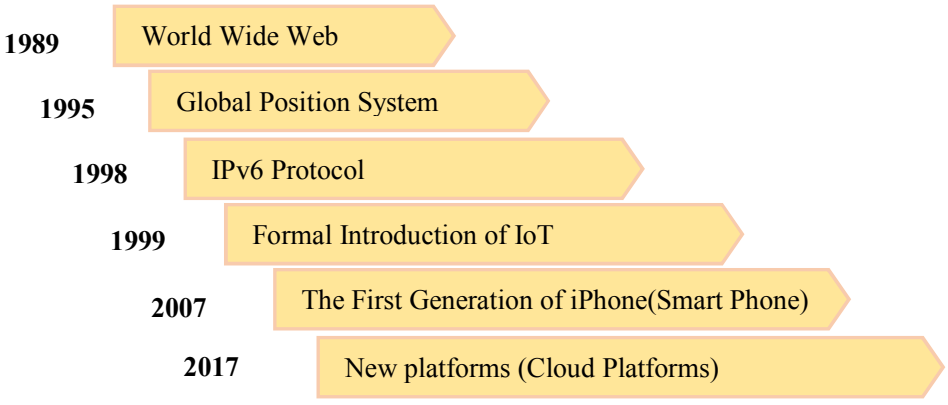

Fig. 1 Some of the most important events in the evolution of IoT 
homes, cities, agriculture, health, and disaster management. Predictions represent the impressive effects of IoT in the near future.

\section{B. Disaster Management}

The term "disaster" is used when the imposed damage exceeds the potential of a community to respond to it and the community requests for the external assistance to overcome the condition. Since a disaster is surprising and gives the respondents a short time to make decisions, responding requires pre-determined planning and strategies and therefore, the term "disaster management" is defined [3]. Disaster management includes a set of actions and attempts made to identify threats, to respond them and to reconstruct a society with disaster. Disaster management can be categorized in four general phases based on the current definitions; risk reduction, preparation, response, recovery [4].

\section{EFFECTS OF IOT ON DISASTER MANAGEMENT}

Technology and specifically, the IoT can recognize and predict the critical situations and crises, prepare for the preconditions and manage the possible post-disaster situations and resources. In this section, the effects of Internet of Things technology on every stages of disaster management have been discussed.

In the risk reduction stage, which must take place before the occurrence of a disaster, embedding sensors in various regions where the potential for danger is high can control the atmospheric and environmental conditions and in the case of danger, prevent many financial and life damages with early warnings and preparations. As an instance, using a temperature, humidity, ultrasonic, gyroscope, or a smoke sensor is able to recognize abnormal temperature, flood, earthquake, and fire respectively by continuous monitoring. In all the mentioned cases, reacting against the existing danger including warning alarms to the residents at these regions and the use of danger mitigation tools such as a fire extinguisher can be performed automatically [5]. Also, space technologies play an undeniable role in accurately visualizing the movements of the Earth in all areas. Satellite images provide a favorable view regarding the disasterprone regions such as earthquake and the Geographic Information System (GIS) helps defining and developing disaster management infrastructures []].

In the preparation stage, using the pre-stored data and their analyses can help creating some models to simulate possible disasters. If some models faster than the real-time models are accessible, it is possible to estimate and predict the damaged areas and the severity of damages even before the prevalence of disaster effects [7]. Simulating the space and location of disaster and using technologies such as the virtual reality have the capacity of increasing the preparation of the critical and important members of the emergency response system and improving disaster management methods [8] as well as training how to deal with the mental conditions and environmental problems in the event of a disaster and how to avoid the physical damages to the public [9].

In the response stage, collecting information about situation is the fundamental and determinant section to satisfy the primary requirements after disaster. Social networks such as Twitter are valuable sources of information for two-sided communications between people and management organizations [10]. the mentioned cases include Cisco's Tactical Operations, a satellite-based telecommunication strategy with the cloud teleology, SERVAL, a technology with the possibility of direct communication of mobile phones even if the network is not covered, and TERA, a text messaging system to make connection between people and relief organizations [11] that all designed to facilitate the communication during disaster. After discussing the data collection, the issue of first aids is raised and inadequate management may lead to financial and human resources losses [9], therefore Felix Wex et al. [12] presented a model for optimizing the scheduling and giving responsibilities to rescue units in disaster situations. $\mathrm{J}$ Sathish Kumar et al. [13] have also presented a real-time model for realizing an applicable method in disaster management operations and rescue plan.

In the recovery stage, IoT can help examining disaster damages and effects, performing the determined recovery plans, and investigating continuously the remaining financial and human resources in compliance with the unresolved damages [14]. For instance, the system proposed by Reza Nourjou et al. [15] is a software for the real-time monitoring of the power outage and its macroeconomic losses whose usage can optimize the energy-related decisions in crises.

Based on what has been stated, the Internet of Things has a considerable potential for developing disaster management that can be converted into a fundamental pattern on the agendas of the relief organizations. This section is condensed in Fig. 2.

This concept can be converted into a fundamental and constant pattern on the agendas of the relief organizations, but it is obvious that using the technology tools requires infrastructures on which IoT can be implemented.

\section{INFRASTRUCTURES REQUIRED FOR USING IOT IN DISASTER MANAGEMENT}

Considering the requirement of implementing the existing plans on a set of devices and protocols, the structure of the Internet of Things technology and then, devices, systems and frameworks used in its implementation are introduced and also, the examples of using them in disaster management systems are mentioned.

\section{A. Layered Architecture of IoT}

Based on different viewpoints, the architecture of the Internet of Things can consist of different numbers of layers whose most basic one is the three layered one including perception layer, middleware layer, and service layer [16]

\section{B. Technology used in each layer}

The technologies required to implement the architecture are as shown briefly in the Table I [17].

\section{Using IoT Architectural Models in Disaster Management}

The existence of a range of the required technologies for each layer with significant structural and physical differences, as some of them mentioned above, allows developers to have many options to improve the performance of their systems. For example, Naser et al. [18] 


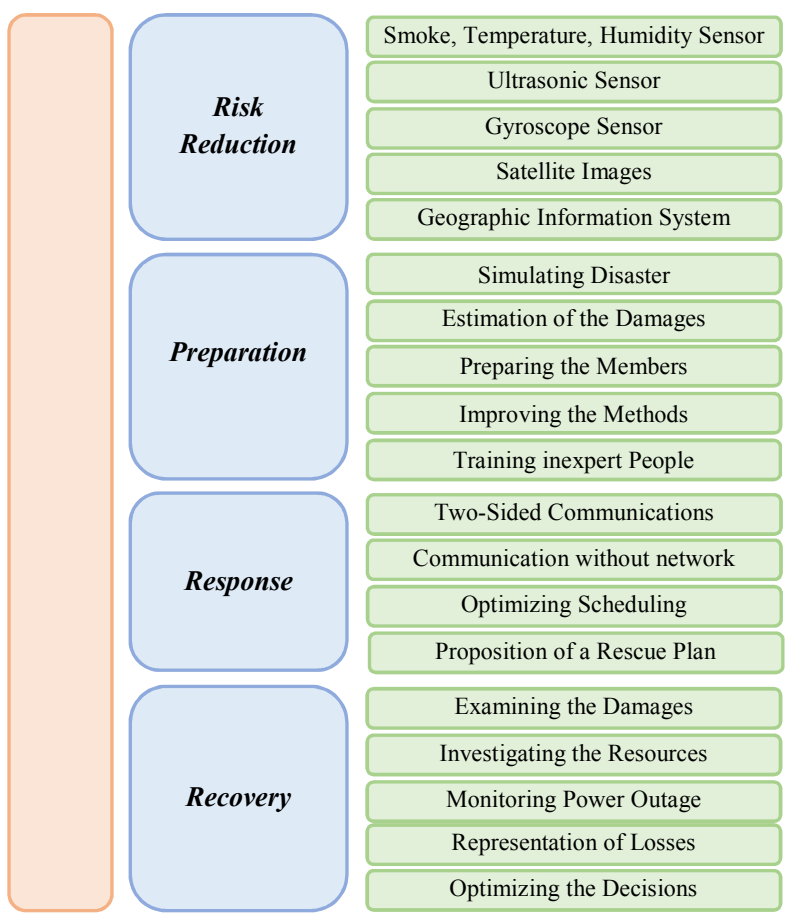

Fig. 2 Effects of IoT on every phase of disaster management

proposed a framework for tracing and representing the key parameters of responding to disasters by adding a cognitive component to an important infrastructure. Infrastructures themselves are disposed to the damage and destruction during crises, therefore the real-time awareness of their status can improve disaster management. In this framework, when a disaster occurs, the collected parameters in the environment are analyzed according to the type of devices embedded in the system either inside the system structure or through the cloud computing and are presented to the relevant authorities in order to have information about the conditions and respond to the infrastructure damage in the best possible manner during a disaster. The three-layer system considered in this framework consists of the first layer: the physical objects and environmental factors, the second layer: a network for collecting, storing and transferring the data to the next layer and the last layer: some programs for examining and further analyzing the data and finally, making decision on the existence of an accident and responding it if required. Also, Talat Butt [19] proposes a comprehensive, intelligent and fog-based scalable architecture which can be implemented for all types of disasters. The architecture presented in this work consists of 5 layers. The data source layer consists of the environmental sensors, RFID, GPS and even social networks; the integration layer consists of infrastructures such as Wi-Fi Direct and LTE D2D; The fog layer consists of the fog networking nodes which can be the deployed and fixed nodes in disaster management system or even devices such as smart digital devices or vehicles which have the fog performance; the cloud layer consists of the traditional centralized cloud infrastructures that include the powerful servers and information repositories; the application layer consists of a variety of applications which can be performed on all lower layers. Other instances are the passive localization systems presented by Gabriel Deak et al. [20] and they use WSN (WoT) IoT in disaster management scenario. Niranjan Kumar Ray et al. [21] proposed a communication framework for the post-disaster situations by improving some effective features such as the network
TABLE I. THE LIST OF REQUIRED DIVICES AND TECHNOLOGIES IN ORDER TO IMPLEMENT IOT

\begin{tabular}{|c|c|c|c|}
\hline $\begin{array}{l}\text { Device / } \\
\text { System }\end{array}$ & Layer & Definition & Pros/Cons \\
\hline Sensors & Perception & $\begin{array}{l}\text { A detector to } \\
\text { measure } \\
\text { environmental } \\
\text { quantities }\end{array}$ & $\begin{array}{l}\text { Diverse, cheep, } \\
\text { Easy to use }\end{array}$ \\
\hline RFID & Perception & $\begin{array}{c}\text { Automated } \\
\text { identification and } \\
\text { information } \\
\text { recording system }\end{array}$ & $\begin{array}{l}\text { Protecting users } \\
\text { with security } \\
\text { protocols }\end{array}$ \\
\hline WSN & Perception & $\begin{array}{c}\text { A group of dispersed } \\
\text { sensors to collect } \\
\text { data }\end{array}$ & $\begin{array}{l}\text { Energy efficient } \\
\text { nodes }\end{array}$ \\
\hline NFC & Perception & $\begin{array}{l}\text { A set of non-contact, } \\
\text { secure and short- } \\
\text { range protocols }\end{array}$ & Low-volume data \\
\hline GPS & Perception & $\begin{array}{l}\text { A global navigation } \\
\text { satellite system }\end{array}$ & $\begin{array}{l}\text { Independent and } \\
\text { unlimited data } \\
\text { transferring }\end{array}$ \\
\hline $\begin{array}{c}\text { Wired } \\
\text { Networks / } \\
\text { Ethernet }\end{array}$ & Middleware & $\begin{array}{c}\text { Physical cables as } \\
\text { media }\end{array}$ & $\begin{array}{l}\text { Economical and } \\
\text { applicable }\end{array}$ \\
\hline $\begin{array}{l}\text { Wireless } \\
\text { Networks }\end{array}$ & Middleware & $\begin{array}{l}\text { Radio frequencies as } \\
\text { media }\end{array}$ & $\begin{array}{l}\text { Further mobility / } \\
\text { less secure and } \\
\text { slower }\end{array}$ \\
\hline $\begin{array}{c}\text { FTTx } \\
\text { Technology }\end{array}$ & Middleware & $\begin{array}{l}\text { Broadband network } \\
\text { architectures using } \\
\text { the optical fiber }\end{array}$ & $\begin{array}{l}\text { Remarkable speed } \\
\text { and quality }\end{array}$ \\
\hline $\begin{array}{c}\text { Wi-Fi } \\
\text { Technology }\end{array}$ & Middleware & $\begin{array}{l}\text { A wireless local } \\
\text { network with a set of } \\
\text { radio technologies }\end{array}$ & $\begin{array}{l}\text { High security and } \\
\text { quality / center- } \\
\text { based } \\
\text { communication }\end{array}$ \\
\hline Bluetooth & Middleware & $\begin{array}{l}\text { Automated, wireless, } \\
\text { short-range } \\
\text { technology standard }\end{array}$ & $\begin{array}{c}\text { Low-cost / } \\
\text { vulnerable to } \\
\text { attacks }\end{array}$ \\
\hline Zigbee & Middleware & $\begin{array}{l}\text { A small wireless } \\
\text { network protocoll }\end{array}$ & $\begin{array}{l}\text { High number of } \\
\text { nodes, low-cost, } \\
\text { low-energy, and } \\
\text { high speed / } \\
\text { security challenges }\end{array}$ \\
\hline $\begin{array}{l}\text { Broadband } \\
\text { Cellular } \\
\text { Network } \\
\end{array}$ & Middleware & $\begin{array}{c}\text { Broadband cellular } \\
\text { network } \\
\text { technologies } \\
\end{array}$ & $\begin{array}{l}\text { Telecommunicatio } \\
\text { n, different speed, } \\
\text { quality, application }\end{array}$ \\
\hline $\begin{array}{c}\text { Cloud } \\
\text { Computing }\end{array}$ & Middleware & $\begin{array}{c}\text { Computing, storage } \\
\text { and applications as } \\
\text { services }\end{array}$ & $\begin{array}{c}\text { Access to data and } \\
\text { services anywhere } \\
\text { and } \\
\text { infrastructures' } \\
\text { cost reduction }\end{array}$ \\
\hline Applications & Service & $\begin{array}{l}\text { Connecting users to } \\
\text { physical devices and } \\
\text { controlling them }\end{array}$ & $\begin{array}{l}\text { Maintainable, wide } \\
\text { range of usage }\end{array}$ \\
\hline
\end{tabular}

power, the network delay and lifetime, which were built on the wireless ad-hoc networks.

\section{EXISTING CHALLENGES}

Although IoT plays a key role in improving disaster management performance, the existing challenges affect the implementation of many plans. In this section, these obstacles will be discussed.

\section{A. Challenges of Disaster Management}

Problems and failures on disaster management are attributed to two general categories: (i) infrastructures and facilities, (ii) human negligence. The essential challenges based on the facilities and infrastructures of disaster management including the high costs of investing on disaster management and inadequate budgets dedicated to this area, old and insecure hospitals [22], inappropriate approach of hospital systems when dealing with disasters [23], weakness of disaster alert systems [24], destruction of the infrastructures during a disaster, communication problems, and existence of a large amount of information [25] create more limitations to examine and respond to disasters. Also, human negligence of both disaster management organizations and people may provide many 
consequences. There is not adequate knowledge and motivation on disaster management, and as a result, the implementation of related programs is not a priority [22]. Current programs are not comprehensive and effective [24]. In addition, there is not enough preparation [23], skill, and awareness of technology [25].

\section{B. Challenges of Disaster Management Using IoT}

As mentioned briefly, there are many challenges to manage disasters and respond to them. In many cases, the IoT comes to help disaster management, but the technology itself has many problems.

Propagation of inaccurate information is possible during a disaster which is intensified by the expansion of the broad communication networks created by IoT. This error may happen due to the error of measurement tools such as the physical nodes of the perception layer, the damage and defect of the network infrastructures or environmental noises. In that case, the process verification of the information will be much more difficult. Therefore, the secure exchange of information [26] is one of the challenges.

Another considerable point is security, whose importance in critical and out-of-control situations will be greater. Security issues in the whole disaster management cycle are very serious challenges as there are many experiences of vulnerability [27] in this regard. Most of these challenges happen because of the inherent features of this technology such as mobility, scalability, heterogeneity, having numerous users under different systems etc. The mobility feature of nodes makes the authentication issue more serious. Also, the extent of networks and out of access points can cause severe security threats as the remote nodes can be manipulated. Because of the heterogeneity of objects securing the communication of objects will be a challenging task [28]. All security threats can be considered as a part or combination of its four main types: the device attacks, the data attacks, attacks to privacy attempts, attacks to network [29]. All types of attacks are conceivable in a disaster situation for misusing the conditions and resources, so they must be addressed to prevent the possible problems

If the security protocols are applied which are an essential principle, some delays will be provided in the system due to the overheads of authentication and information verification [30] which have a significant effect on responding disaster at high scales with high volume of nodes and information, especially when the time is a key factor during a disaster. Therefore, more advanced techniques should be implemented to optimize the networks and communications.

All above-mentioned points are summarized in Fig. 3.

\section{RESEARCHES ON IMPLEMENTATION OF IOT SYSTEMS FOR DISASTER MANAGEMENT}

In this section, some instances of the IoT-based actions taken to manage three deadly and frequent disasters i.e. earthquake, flood, and fire are introduced.

\section{A. Disaster Management and Earthquake}

Since there is not any solution to predict and prevent the earthquake, the best action during an earthquake is early

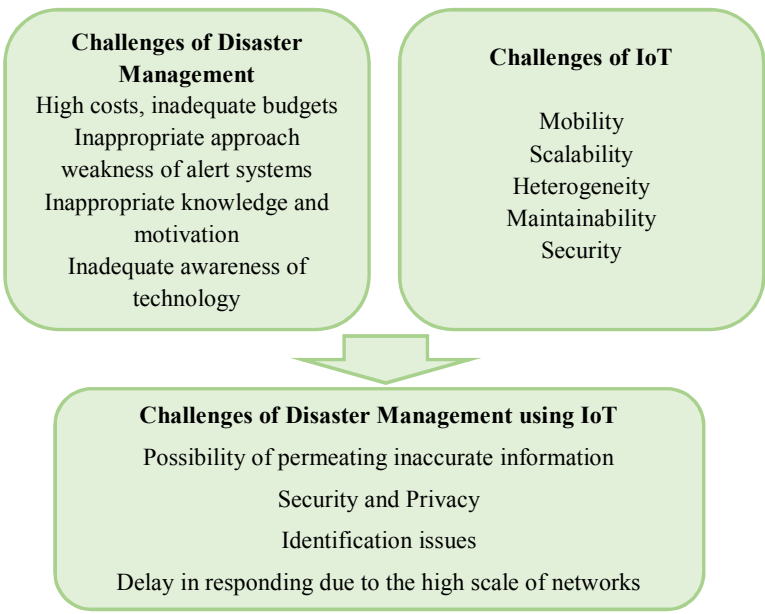

Fig. 3 Challenges of disaster management, IoT, and disaster management using IoT

warning based on the foreshocks to reduce the losses and this can be implemented automatically using IoT.

The system designed by Allu Suresh Babu et al. [31] uses the small shocks of the earth to detect the fundamental vibrations and then, generates and sends the alert messages when an earthquake is detected. In this system, NodeMCU platform, the Lua programming language, and M2M (IoT) connection protocol are used. The GY-61 DXL335 accelerometer sensor module is also used to detect the dynamic acceleration. Cayyenne software is also used to generate and send the alerts.

The instrumentation system designed by Suma M et al. [32] detects the foreshocks using IoT technology. The set of components and devices used in this system include a piezoelectric accelerometer, an ADC 809 converter, a P89V51RD2 microcontroller, a type of $64 \mathrm{kB}$ flash memory and a 1024 bytes RAM, a vibration sensor, GSM, and LCD. the platform uses 8051 and $\mathrm{C}$ programming language.

The system designed by $\mathrm{N} \mathrm{N}$ Venkatesh Gupta et al. [33] senses the conditions in the real-time form, updates the data, and activates the alarm systems if an earthquake is detected. In this system, NodeMCU microcontroller is used as the processing core. The sensors used in this system are the type of micro-electromechanical systems (MEMS). Thinkspeak is the IoT-based open source platform of this system.

The developed earthquake warning system designed by Abdulkadir Karaki [34] detects the non-destructive foreshocks, publishes some information on Twitter about the beginning and ending time of earthquake and also warns residents. The system uses Arduino Mega microcontroller, IMU sensor and Grove-Piezo vibration sensor card which are capable of detecting vibrations independently. Also, ESP8266 WiFi module and a buzzer are used. System's platform is the combination of processing and wiring language.

The early warning system designed by A.M. Zambrano et al. [35] can predict the maximum earthquake in the center of earthquake up to 12 seconds using smartphones. The system was designed in three layers. smartphone nodes are used instead of microcontrollers. The devices of the system are as follows: WiFi 2G/3G / 4G networks and sensor web. 
The protocols used in the system are MQTT, Network Time Protocol (NTP) and a secure socket layer (SSL).

\section{B. Disaster Management and Flood}

In addition to the possibility of warning when facing a flood, considering the nature of this phenomenon, it is possible to predict and take actions before its occurrence.

The model proposed by Massimo Ancona et al. [36] is a model of flash flood monitoring system and its related warning system. In this model, the previous systems have been optimized by IoT platforms. The perception layer in this model can be implemented using the intelligent wireless sensor network consisting of only rain gauges or river gauges, or both. High energy consumption problem can be solved by creating a protective layer for each node; in addition to the sensor, an ultra-low-power microcontroller, a buck converter, a boost charger, and a Texas Instrument CC3100 are needed. In this system, all platforms designed for IoT can be used. The named platforms include: Axeda, Thingworx, M2M Labs, Arduino, etc.

The system proposed by Wahida Md. Shah et al. [37] can approximate and inform the time of flood occurrence by controlling the water level using two sensors at two different levels and the speed of rising water level. The system uses cascade model. The SEN113104 sensor, GSM module, USB $3 \mathrm{G}$ modem Huawei mobile broadband, and Raspberry Pi are parts of the system. System uses python language.

Sandeep K. Sood et al. [38] propose a flood forecasting and monitoring architecture based on IoT and HPC and big data convergence. In this architecture, there are 4 layers of capability to collect, store and analyze big data through HPC, but the information is collected by IoT devices. The layout models of IoT devices are considered to be hexagons for optimality. Data is obtained through social networks, databases, and a variety of water level, waterflow, and rainfall sensors. The model uses raspbery pi, virtual switch software as fog layer, and amazon elastic computing cloud as data analysis layer.

The system proposed by Alisson Slva Suza et al. [39] is able to warn the emergency situation using the symbolic lights and to inform the responsible centers using the short message system and phone calls in order to reduce the damage in urban centers. The sensor is HC-SR04, the microcontroller is ESP8266 NodeMCU and the communication module is GSM. Cloud and Ubidots platforms store information.

The method proposed by Farzad Alamdar et al. [40], can evaluate qualitatively the activities of involved components, access, exchange and use the large-scale data in real environments. In this paper, the problems existed on the use of sensor data for disaster management in the interorganizational relationships are explored and the most prominent of which are as follows: Lack of using the existing information, Being limited in supporting the sensor systems, Need to change manually the data interface, Limitations of utilizing the usable capacity of resources, Lack of an integrated information model, Incompatibility and heterogeneity in the data, Lack of a common language.

\section{Disaster Management and Fire}

Fire has different types and it can be classified based on the cause and location. Forecasting, warning, guiding and tracking people and equipment are possible actions to reduce the number of losses in fires. Automation can increase the efficiency.

In researches provided by Zhang Ying-cong et al. [41], the suggestions on IoT applications in this regard are as follows: By equipping the firefighting support tools with separate unique identity codes, their status are traceable, By monitoring the quality of firefighting tools in all stages of their lifecycle their lifetimes increase dramatically, By equipping buildings with the automatic fire extinguishing systems, the safety against the fire is increased, by making homes smart, there is the possibility of remote monitoring of the situation, using IoT, if a fire happens, information is provided in a coherent and real-time form.

The system designed by Huixian Jiang [42] uses AI algorithms and IoT equipment to control buildings and develop an evacuation route model for the safe exit of people from public buildings in the fire. If a fire occurs, the warning bells will make an alarm sound. in addition to showing a safe exit route in real-time, system makes emergency calls to the close firefighting units. RFID labels, laser rangefinders, and fire-sensitive sensors as well as ArcGIS Android SDK 10.1 platform are parts of the system.

In the framework proposed by Harkiran Kaur et al. [43], IoT, cloud computing and fog computing are used to predict and alarm forest fires. Temperature, rainfall, wind speed, and humidity sensors as well as the database information on the climate status such as drought are used in the data collection layer. In this system, the computing nodes are smartphones. Cloud layer and computations are covered by Amazon Elastic Compute Cloud. The fog layer is implemented on the nodes as an application based on Javascript.

The structured fire visualization and warning system designed by Xiu-Shan Chen et al. [44] combines three research areas of IoT, Fire Dynamics Simulation, and Building Information Modeling to provide 4 types of services for disaster management: fire visualization, monitoring the environment, disaster warning, guiding people. These services increase the awareness of firefighter and reduce the fear and anxiety of people inside the building. The role of the IoT is to collect information and control the signs. Grove Flame sensors, Carbon Monoxide concentration and LED MAX7218 are placed in the real space. The system uses Autodesk Revit to implement BIM, CAD method to exchange and translate the data, and PyroSim to forecast fire components.

In the studies performed by Yu-xi Guan et al. [45], an emergency rescue system has also been simulated using ZigBee IoT so that the fire is detected using thermal imaging. The information about situation is sent to the firefighting centers and hydrant fire extinguishing system is activated. Firefighters can find the best path to reach the event location. Other capabilities of the system include disaster warning facility and contacting relevant authorities. RF cards, temperature, humidity and gas concentration sensors, GPS, and radio frequency positioning system are used.

The summery of three last subsections are presented in Table II. 
TABLE II. THE SKETCH OF INSTANCES OF APPLICATION OF IOT IN TREE DISASTERS

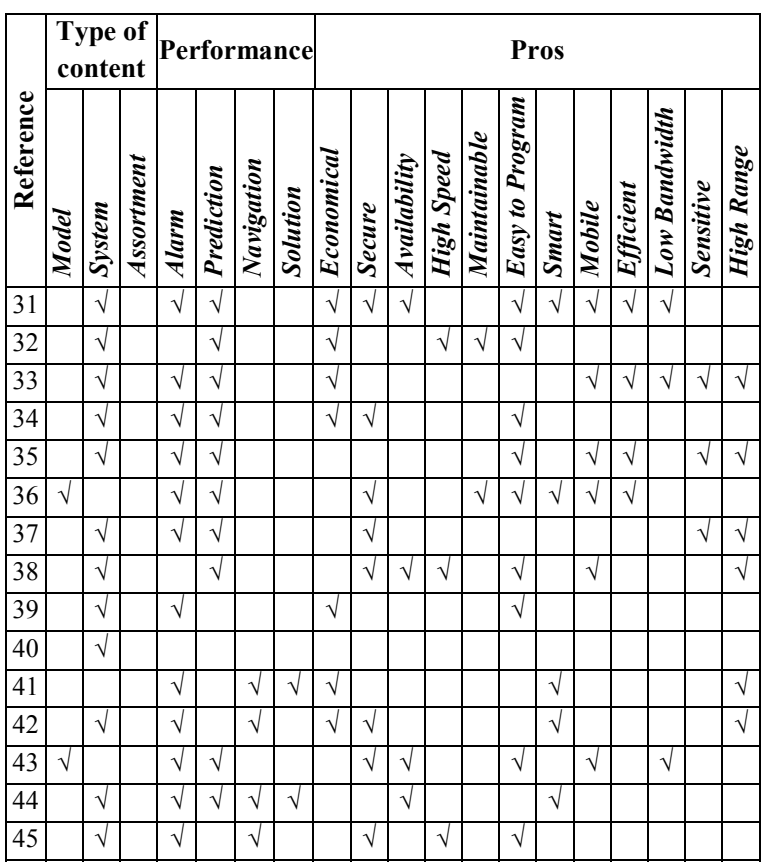

\section{CONCLUSION AND FUTURE WORKS}

Using IoT in disaster management has become a major concern in recent years, therefore a large number of systems and models have been presented to detect the risks of disasters or offer practical solutions to them with the objective of saving lives and reducing damages. Many researches have been provided in this area each of which deals with specific issues. What is proposed in this paper is a comprehensive overview of the positive and negative aspects of this application with novel approaches. In the approach of examining different phases of disaster management, the kinds of tasks that IoT can do in each phase were discussed by presenting some instances of previous systems and models. In the approach of analyzing the required infrastructure, it was determined that most of the protocols and devices required to implement the intelligent IoT-based disaster management systems are also in our daily lives and they are accessible and in some cases they are even cheap, therefore, we do not have much restriction in this regard. In the approach of examining the available challenges, the kind of problems that disaster management faces were determined some of which can be solved using IoT. Also, it was found that using IoT itself creates some problems that they have also been discussed. In the approach of examining the use of IoT in different types of disasters, three disasters, earthquake, flood and fire were selected and analyzed by introducing the previous systems in these fields and finally, it was determined that: (i) In the field of earthquake, investments focus mostly on foreshock based warning systems, (ii) In the field of flood, in addition to warning, it can be predicted. Databases and the analysis of environmental status records are used in the management in addition to controlling the environmental parameters, (iii) In the field of fire, in addition to warning and forecasting, some systems and models are implemented to find the route and guide public and rescuers as well as tracing the tools and equipment. Our future works can include investigating the application of IoT in other disasters including other environmental disasters such as tsunamis and human-made disasters such as the pollution of water resources.

\section{REFERENCES}

[1] "Internet of Things (IoT) for effective disaster management", Digital India Action Group.

[2] Qin Wang, Xinqi Zhu, Yiyang Ni, Li Gu, Hongbo Zhu, "Blockchain for the IoT and industrial IoT: A review", Journal of Internet of Things, 2019.

[3] www.un-spider.org

[4] Nadia Saad Noori, Christina Weber, "Dynamics of coordinationclusters in long-term rehabilitation", Journal of Humanitarian Logistics and Supply Chain Management, 2016, Vol(6), pp 296- 328.

[5] Soubhagyalaxmi D B, Sushma L Bhosle, Swetha T, Veena P D, Chetan B V, "Disaster management system using IoT", Journal of IJRTI, vol (3), 2018, pp 148-154.

[6] Kazuya Kaku, "Satellite remote sensing for disaster management support: A holistic and staged approach based on case studies in Sentinel Asia”, International Journal of Disaster Risk Reduction, Vol (33), 2019, pp 417-432.

[7] Ujjwal K.C., Saurabh Garg, James Hilton, Jagannath Aryal, Nicholas Forbes-Smith, "Cloud Computing in natural hazard modeling systems: Current research trends and future directions", International Journal of Disaster Risk Reduction, vol (38), 2019.

[8] Francesco Longo, Letizia Nicoletti, Antonio Padovano, "Emergency preparedness in industrial plants: A forward-looking solution based on industry 4.0 enabling technology", Journal of Computers in Industry, vol (105), 2019, pp 99-122.

[9] Mihoko Sakurai, Yuko Murayama, "Information technologies and disaster management - Benefits and issues", Journal of Progress in Disaster Science, vol (2), 2019.

[10] Nastaran Pourebrahim, Selima Sultana, John Edwards, Amanda Gochanour, Somya Mohanty, "Underestanding communication dynamics on twitter during natural disasters: A case study of Hurricane Sandy", International Journal of Disaster Risk Reduction, vol (37), 2019.

[11] safetymanagement.eku.edu

[12] Felix Wex, Guido Schryen, Stefan Feuerriegel, Dirk Neumann, "Emergency response in disaster management: Allocation and scheduling of rescue units", vol (3), 2014, pp 697-708.

[13] J Sathish Kumar, Mukesh A Zaveri, Meghavi Choksi, "Task based resource scheduling in IoT environment for disaster management", Journal of Procedia Computer Science, vol (115), 2017, pp 846-852.

[14] "Strengthening the disaster resilience of the academic biomedical research community: protecting the nation's investment.", National Academies Press, 2017.

[15] Reza Nourjou, Mehdi Hashemipour, "Smart energy utilities based on real-time GIS web services and IoT", Journal of Procedia Computer Science, vol (110), 2017, pp 8-15.

[16] Anju S. Pillai, Gaddam S. Chandraprasad, Ahmed S. Khwaja, Alagan Anpalagan, "A service oriented IoT architecture for disaster preparedness and forcasting system", Journal of Internet of Things, 2019.

[17] Imed Romdhani, Riad Abdmeziem, D. Tandjaoui, "Architecting the Internet of Things: State of the Art", 2015.

[18] Naser M. Z., Kodur V.K.R., "Cognitive inftastructure - a modern concept for resilient performance under extreme events", Journal of Automation in construction, 2018, vol(90), pp 253-264.

[19] Talal Ashraf Butt, Context-aware Cognitive Disaster Management using Fog-based Internet of Things, 2019.

[20] Gabriel Deak, Kevin Curran, Joan Condell, Eleana Asimakopoulou, Nik Bessis, "IoTs (Internet of Things) and DfPL (Device-free Passive Localisation) in a disaster management scenario", Journal of Simulation Modelling Practice and Theory, 2013, vol(35), pp 86-96.

[21] Niranjan Kumar Ray, Ashok Kumar Turuk, "A framework for postdisaster communication using wireless and hoc networks", Journal of Integration, 2017, vol(58), pp 274-285.

[22] Masoumi GhR, Dehghani A., "Challenges of implementing disaster preparedness plan in Iran hospitals", Journal of Health in Emergencies and Disasters Quarterly, vol (2), 2016, pp 3-4.

[23] Sima Feizolahzadeh, Ali Akbar Vaezi, Masoud Mirzaei, Hamidreza Khankeh, Ali Taheriniya, Mohammadreza Vafaeenasab, Davoud Khorasani-Zavareh, "Barriers and facilitators to provide continuity of care to dischargeable patients in disaster: A Qualitative study", Journal of Injury, vol (50), 2019, pp 869-876. 
[24] Japhet Biadoo, "Challenges and Strategies for Rapid Response in Disaster Relief Operations in Ghana", Texila International Journal of Management, vol (4), 2018.

[25] Billy Haworth, "Emergency management perspectives on volunteered geographic information: Opportunities, challenges and change", Journal of Computers, Environment and Urban Systems, vol (57), 2016, pp 189-198.

[26] Muhammad Ibrahim Channa, Kazi M. Ahmed, "emergency response communications and associated security challenges", vol (4), 2010.

[27] Alem Colakovic, Mesud Hadzialic, "IoT: A review of enabling technologies, challenges, and open research issues", Journal of Computer Networks, vol (144), 2018, pp 17-39.

[28] Chakib Bekara, "Security Issue and Challenges for the IoT-based Smart Grid", Journal of Procedia Computer Science, vol (34), 2014, pp 532-537.

[29] Kenneth Kimani,Vitalice Oduol,Kibet Langat, "Cyber security challenges for IoT-based smart grid networks", Journal of critical infrastructure protection, vol (25), 2019, pp 36-49.

[30] Abderazek Seba, Nadia Nouali-Taboudjemat, Nadjib Badache, Hamida Seba, "A review on security challenges of wireless communication in disaster emergency response and disaster management situation", Journal of Network and Computer Applications, vol (126), 2019, pp 150-161.

[31] Allu Suresh Babu, Prof.G.T.Naidu, U.Meenakshi, "Earth Quake Detection and Alerting Using Iot", International Journal of Engineering Science Invention, vol (7), 2018, pp 14-18.

[32] Suma M, Sushma S V, Swathi S Poojari, Sanjana R, Prof. Kiran M, "A novel technique for automatic detection of earthquake and landslide using IoT", Journal of Computing, Communications and Networking, vol (7), 2018, pp 160-163.

[33] N N Venkatesh Gupta, Mr. S. Rinesh, "IoT based earthquake detection by thigspeak", vol (119), 2018, PP 3089-3095.

[34] Abdulkadir Karaci, "IoT-based earthquake warning system development and evaluation", Mugla Journal of Science and Technology, vol (4), 2018, pp 156-161.

[35] A.M. Zambrano, I.Perez, C. Palau, M. Esteve, "Technologies of Internet of Things applied to an Earthquake Early Warning System”, 2017, vol(75), pp 206-215.

[36] Massimo Ancona, Andrea Dellacasa, Giorgio Delzanno, Andrea La Camera' Ivano Rellini, “An "Internet of Things” Vision of the Flood Monitoring Problem", Conference on ambient computing, applications, services and technologies, 2015, pp 26-29.

[37] Wahidah Md. Shah, F. Arif, A.A. Shahrin, Aslinda Hassan, "The Implementation of an IoT-Based Flood Alert System", International Journal of Advanced Computer Science and Applications, vol (9), 2018, pp 620-623.

[38] Sandeep K. Sood, Rajinder Sandhu, Karan Singla, Victor Chang, "IoT, big data and HPC based smart flood management framework", Journal of Sustainable Computing: Informatics and Systems, vol (20), 2018, pp 102-117.

[39] Alisson Silva Souza, Andre Marcio de Lima Curvello, Flavio Luiz dos Santos de Souza, "A flood warning system to critical region", Journal of Procedia Computer Science, vol (109), 2017, pp 11041109.

[40] Farzad Alamdar, Mohsen Kalantari, Abbas Rajabifard, "Understanding the provision of multi-agency sensor information in disaster management: A case study on the Australian state of Victoria”, Journal of Disaster Risk Reduction, vol (22), 2017, pp 475493.

[41] ZHANG Ying-cong, YU Jing, "A Study on the Fire IOT Development Strategy", Journal of Procedia Engineering, vol (52), 2013, pp 314-319.

[42] Huixian Jiang, "Mobile Fire Evacuation System for Large Public Building Based on Artificial Intelligence and IoT", Journal of IEEE, vol (7), 2019, pp 64101-64109.

[43] Harkiran Kaur, Sandeep K. Sood, "Fog-assisted IoT-enabled scalable network infrastructure for wildfire surveillance", Journal of Network and Computer Applications, vol (144), 2019, pp 171-183.

[44] Xiu-Shan Chen, Chi-Chang Liu, I-Chen Wu, "A BIM-based visualization and warning system for fire rescue", Journal of Advanced Engineering Informatics, vol (37), 2018, pp 42-53.

[45] Yu-xi GUAN, Zheng FANG, Tian-ran WANG, "Fire Risk Assessment and Daily Maintenance Management of Cultural Relic Buildings Based on ZigBee Technology", Journal of Procedia Engineering, vol (211), 2018, pp 192-198. 